\title{
Dynamical QCD+QED simulation with staggered quarks
}

\section{Ran Zhou*}

Department of Physics, Indiana University, Bloomington, IN 47405, USA

Theoretical Physics Department, Fermi National Accelerator Laboratory, Batavia, 60510, USA ${ }^{\dagger}$

E-mail: zhouranefnal.gov

\section{Steven Gottlieb}

Department of Physics, Indiana University, Bloomington, IN 47405, USA

\section{MILC Collaboration}

Electromagnetic effects play an important role in many phenomena such as isospin-symmetry breaking in the hadron spectrum and the hadronic contributions to g-2. We have generalized the MILC QCD code to include the electromagnetic field. In this work, we focus on simulations including charged sea quarks using the RHMC algorithm. We show details of the dynamical QCD+QED simulation algorithm with compact QED. We analyze the code performance and results for hadron-spectrum observables.

The 32nd International Symposium on Lattice Field Theory,

23-28 June, 2014

Columbia University New York, $N Y$

* Speaker.

$\dagger$ present address 


\section{Introduction}

Electromagnetic corrections to phenomena dominated by the strong interaction have received considerable attention in recent years. For example, the electromagnetic effect on the hadron mass spectrum has been investigated extensively in lattice simulations [1-6]. Although there has been much progress in this area, much of the research relies upon the quenched QED approximation that neglects sea-quark charges. There are some difficulties in estimating systematic errors associated with the quenched-QED approximation. Further, sea-quark electromagnetic effects are critical in some studies. For example, the sea-quark electromagnetic interaction contributes a term to the pseudoscalar meson mass, and one of the Low Energy Constants (LECs) in meson QCD+QED chiral perturbation theory (ChPT) is associated with this term. To determine this LEC in a quenchedQED simulation, one has to use reweighing $[7,8]$. In addition, the electromagnetic effect is also the dominant systematic in the current $m_{u} / m_{d}$ determination. Similarly, sea-quark electromagnetic effects also play a role in the lattice-QCD muon anomalous magnetic moment calculation [9]. The quark-disconnected contribution, for instance, is a significant source of the systematic error in the current calculations of the hadronic light-by-light term. The sea quarks are coupled to the electromagnetic field, and the sea-quark electromagnetic effect is not negligible.

Fully dynamical QCD+QED calculations include the $U(1)$ photon field during configuration generation. This approach eliminates the unquantifiable quenching error. Recently, the QCDSF [10] and BMW [11] collaborations presented their dynamical QCD+QED studies of the meson and baryon masses based upon the non-compact QED formalism. In this work, we report on our progress developing a QCD+QED simulation code based on the compact QED formalism.

The structure of this paper is as follows. We describe the details of the numerical algorithm of the dynamical QCD+QED simulation in Sec. 2. We then show various test results related to configuration generation and the pseudoscalar meson spectrum in Sec. 3. Finally, we conclude by summarizing our progress and briefly considering future steps.

\section{Methodology}

\subsection{Non-compact QED and compact QED}

There are two methods for including the electromagnetic field in the lattice simulations. Noncompact QED employs an electromagnetic gauge potential $A_{\mu}$ whose value ranges from $-\infty$ to $\infty$. The QED action is calculated from the gauge potential similarly to the way it is in the continuum.

$$
S_{\mathrm{QED}}=\frac{1}{4} \sum_{x, \mu, v}\left(\partial_{\mu} A_{v}(x)-\partial_{v} A_{\mu}(x)\right)^{2},
$$

but the derivative is replaced by a finite-difference approximation. The advantage of this method is that in quenched-QED calculations, the $U(1)$ configurations are generated independently without using a Markov chain. One can generate $A_{\mu}$ in momentum space from a Gaussian distribution and Fourier transform the results back to coordinate space. Therefore, there are no autocorrelations among the $U(1)$ configurations. However, this is not true in dynamical QCD+QED simulations, although there are some techniques to decrease the autocorrelations between consecutive $U(1)$ 
configurations [11]. In addition, the non-compact $U(1)$ formalism requires gauge fixing during the $U(1)$ gauge-field generation process.

The other method, the compact QED formalism, uses a complex number to represent the lattice $U(1)$ field. The QED action is written as

$$
S_{\mathrm{QED}}=\beta \sum_{x, \mu, v}\left(1-\square_{\mu v}\right),
$$

where the $\beta=1 / e^{2}$ and $\square_{\mu \nu}$ is the $U(1)$ plaquette. The compact-QED action is quite similar to the Wilson QCD action, which allows us to reuse the preexisting $S U(3)$ code with minimum change (but loss of efficiency). In addition, the $U(1)$ gauge fixing can be done after configurations are generated, which makes it somewhat easier to implement the dynamical QCD+QED algorithm. Because of these advantages of the compact QED, we implemented our dynamical QCD+QED algorithm with the compact QED formalism. However, it should be kept in mind that the compact $U(1)$ formalism has photon self-interactions as a lattice artifact, and we must treat this artifact carefully in our analysis.

\subsection{Dynamical QCD+QED simulation algorithm}

We start this section with a brief recap of the RHMC algorithm for dynamical QCD simulations. We then extend the algorithm to dynamical QCD+QED. We follow the notation in Ref. [12]. In continuum QCD, the expectation value of an observable $\hat{O}$ is given by a path integral:

$$
\langle\hat{O}\rangle=\frac{1}{Z(\beta)} \int \prod_{x, \mu} d U_{\mu}(x) \hat{O}\left(\operatorname{det} M_{F}\right)^{n} \exp \left\{-S_{G}\right\}
$$

where the fermion fields are integrated out resulting in a fermion determinant $\operatorname{det} M_{F}$ (raised to an appropriate power) and $S_{G}$ is the gluon-field action. We generate the $S U(3)$ gluon field $U_{\mu}$ with probability distribution $P_{U}$ using effective action $S_{\text {eff: }}$ :

$$
\begin{aligned}
P_{U} & =\frac{1}{Z(\beta)}\left[\operatorname{det} M_{F}(U)\right]^{n} \exp \left\{-S_{G}(U)\right\}=\frac{1}{Z} \exp \left\{-S_{\mathrm{eff}}(U)\right\}, \\
S_{\mathrm{eff}} & =S_{G}(U)+n \operatorname{Tr} \ln M_{F}(U) .
\end{aligned}
$$

Using hybrid molecular dynamics, one adds a conjugate momentum dependent term to $S_{\text {eff }}$ to form an effective Hamiltonian [13]

$$
H(p, U)=\sum_{x, \mu} \frac{1}{2} \operatorname{Tr} H_{\mu}^{2}+S_{\mathrm{eff}}(U),
$$

where $H_{\mu}$ is a traceless Hermitian matrix. The evolution of the system is given by Hamilton's equations,

$$
\left\{\begin{array}{l}
\dot{U}_{\mu}=i H_{\mu} U_{\mu} \\
\dot{H}_{\mu}=\left.i U_{\mu} \frac{\partial S_{\mathrm{eff}}}{\partial U_{\mu}}\right|_{\mathrm{TH}},
\end{array}\right.
$$

where TH means a traceless Hermitian projection. The $\operatorname{Tr} \ln M_{F}(U)$ is handled by introducing a pseudo-fermion field $\Phi$, but now we must deal with the inverse of $M_{F}(U)$.

$$
S_{\mathrm{eff}}=S_{G}(U)+\Phi^{+} M_{F}^{-1} \Phi,
$$


and the force becomes

$$
\frac{\partial S_{\mathrm{eff}}}{\partial U_{\mu}}=\frac{\partial S_{G}}{\partial U_{\mu}}-\left.\Phi^{+} M_{F}^{-1}(U) \frac{\partial M_{F}(U)}{\partial U_{\mu}} M_{F}^{-1}(U) \Phi\right|_{\mathrm{TH}} .
$$

The two terms on the RHS are the gauge force and fermion force, respectively. Both are needed to update the conjugate momentum.

To incorporate QED into the dynamical QCD simulation and obtain a dynamical QCD+QED algorithm, we need to implement several changes. We add $U(1)$ field links $\left(U_{\mu}^{\mathrm{QED}}\right)$ and corresponding conjugate-momentum variables. We then add a QED gauge contribution to the action,

$$
S=S_{G}^{\mathrm{QCD}}+S_{G}^{\mathrm{QED}}+\Phi^{+} M_{F}^{-1}(U) \Phi,
$$

where the fermion matrix $M_{F}$ includes both QCD and QED effects. We add code to update the $U_{1}$ field $\left(U_{\mu}^{\mathrm{QED}}\right)$ and its conjugate momentum according to Hamilton's equations. Finally, we change the QCD fermion force to take into account the quark electromagnetic interactions and introduce the new QED fermion force. In dynamical QCD+QED simulations, the sea quarks carry both $S U(3)$ and $U(1)$ charges. The QCD code we started with allowed different staggered fermion actions including one link, one link plus Naik term, asqtad, and HISQ. Currently, we combine the $S U(3)$ and $U(1)$ links and then smear the combined link. That is, for each charge we calculate $U_{\mu}^{Q E D}(x)$ for that charge and then apply the desired smearing routine to

$$
U_{\mu}(x)=U_{\mu}^{Q C D}(x) U_{\mu}^{Q E D}(x),
$$

This method has been used in our quenched-QED simulations $[3,4]$ and works well. An alternative is to separately smear the $S U(3)$ and $U(1)$ links, which would require some more code development. In light of our prior success with the former method, we stick with it for the dynamical QCD+QED simulation. Hamilton's equations for the QED field and momentum are

$$
\begin{aligned}
\dot{U}_{\mu}^{Q E D} & =i H_{\mu}^{Q E D}(x) U_{\mu}^{Q E D}(x), \\
\dot{H}_{\mu}^{Q E D} & =i U_{\mu}^{Q E D}(x) \frac{\partial S_{\mathrm{eff}}(U)}{\partial U_{\mu}^{Q E D}}=\operatorname{Tr}\left[i U_{\mu}(x) \frac{\partial S_{\mathrm{eff}}(U)}{\partial U_{\mu}}\right],
\end{aligned}
$$

where the trace is over $S U(3)$ indices. Comparing with Hamilton's equations of $\dot{H}_{\mu}^{Q C D}$, we take the projection of trace of the force term. In addition, the second equal sign in the second equation comes from both the chain rule and Eq. (2.11), which is another reason why we use Eq. (2.11). Allowing independent smearings for QCD and QED gauge fields would require additional code development.

\section{Results}

We implemented the new features required for dynamical QCD+QED simulation algorithm starting from the MILC dynamical-QCD simulation code. To check the new code, we studied how well the effective Hamiltonian is conserved. In the MILC code, we can choose different integrators such as leapfrog, Omelyan, etc. We first tested the leapfrog integrator. On a $6^{4}$ grid, starting from fixed random $S U(3)$ and $U(1)$ fields, we ran the leapfrog algorithm with varying step sizes, but 


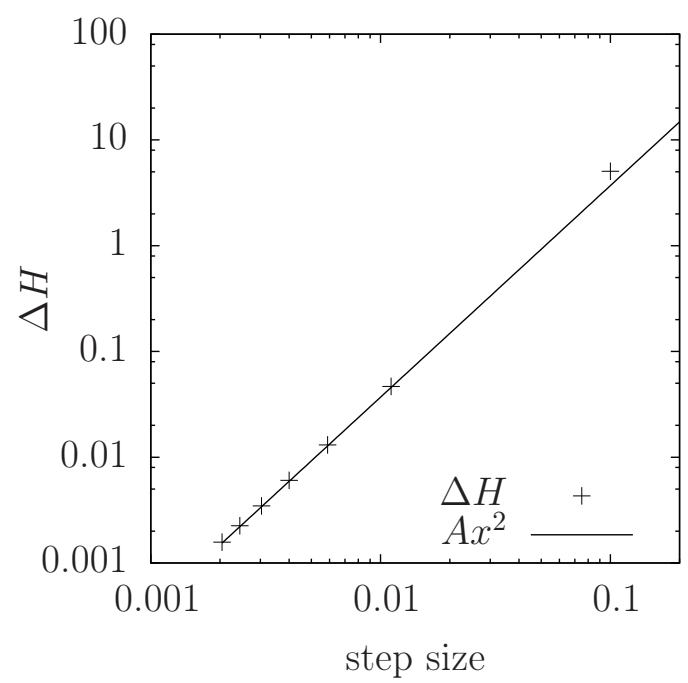

Figure 1: Test of conservation of the Hamiltonian with the leapfrog integrator in the dynamical QCD+QED code. The line is proportional to the square of the step size, showing that $\Delta H$ has the expected behavior. The slope of the line indicates that $\Delta H$ is proportional to the square of the step size.

fixed unit total trajectory length. Figure 1 shows that the change in the effective Hamiltonian $\Delta H$ is proportional to the square of the step size, as expected. We varied the integrator and starting configuration to verify expected behavior in several additional cases.

After we confirmed that the integrator works correctly, we started a test run on a $6^{4}$ lattice with $\beta_{Q C D}=5.5$ and $\beta_{Q E D}=10.0$. All of the sea quarks have the same electromagnetic charge. We plot the evolution of the average $S U(3)$ and $U(1)$ plaquettes in Fig. 2. Both of the QCD and QED field started from free-field configurations. In the left panel, we observe that the $S U(3)$ field has a different equilibrium time from the $U(1)$ field. The right panel shows enlarged plots of the evolution of the $S U(3)$ (right-upper) and $U(1)$ (right-lower) fields. The black line denotes the theoretical prediction for the average $U(1)$ plaquette in the weak coupling limit. Our test shows that the evolution of the configuration is consistent with theoretical expectations. Similar results have been obtained in Ref. [14].

The electromagnetic contribution to the pseudoscalar-meson mass has been studied extensively in quenched QED. We generated a test ensemble with size of $12^{3} \times 32$. All three sea quarks have the same mass, 0.029 in lattice units. We set $\beta_{\mathrm{QCD}}=6.76$ and $\beta_{\mathrm{QED}}$ at its physical value. We used the unimproved staggered quark action in this test. We calculated the pseudoscalar meson mass for a few values of the valence quark charge. The meson, composed of a quark and anti-quark is neutral, but its mass depends on value of the (anti-)quark charge. We extract the electromagnetic contribution to the meson mass via:

$$
\delta m^{2}=m^{2}\left(e^{\mathrm{val}} \neq 0\right)-m^{2}\left(e^{\mathrm{val}}=0\right) .
$$

Chiral perturbation theory for QCD+QED predicts that to leading order $\delta m^{2}$ is proportional to $\alpha_{\mathrm{EM}}^{\mathrm{val}}[15]$. Our calculation is consistent with this. 

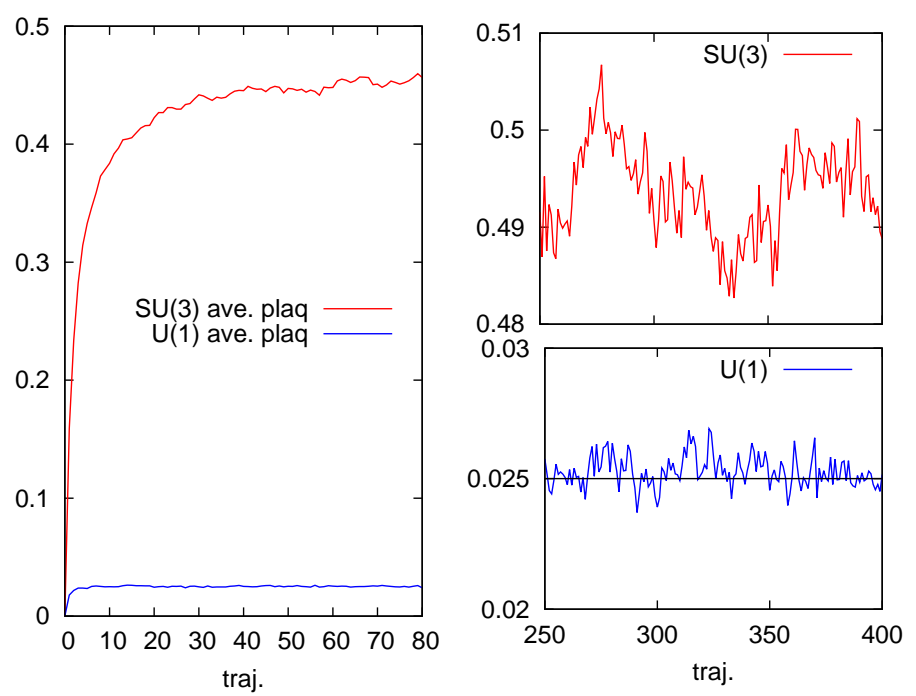

Figure 2: Test of the evolution of the averaged plaquette from the dynamical QCD+QED gauge code. Our result is consistent with the prediction.

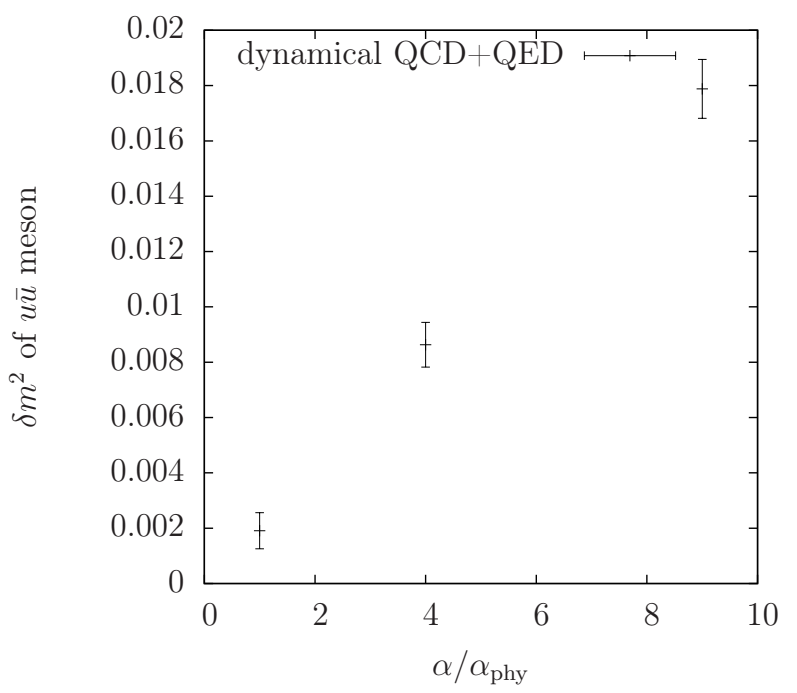

Figure 3: The $\delta m^{2}$ calculated from different valance electromagnetic charges. The result is proportional to $\alpha_{\mathrm{EM}}^{\mathrm{val}}$ as expectated from chiral perturbation theory.

\section{Summary and outlook}

In this work, we discuss the RHMC algorithm for dynamical QCD+QED calculations with compact QED. We implemented the algorithm based upon MILC dynamical-QCD code. We tested the correctness and performance of our code by checking the discretization error of the integrator, the evolution of the plaquette, and the electromagnetic contribution to the pseudoscalar-meson mass. These tests show the expected behavior and give us some confidence to generate additional QCD+QED ensembles with larger lattice sizes and multiple sea-quark mass and charge combinations. We have also recently begun comparing our results with an independent coding effort of 
James Osborn based on FUEL. Eventually, this will allow us to perform high-precision calculations that include electromagnetic effects.

Acknowledgments: R. Z. thanks Thomas Blum for helpful discussions about the dynamical QCD + QED algorithm when he was a student at the University of Connecticut. We thank Claude Bernard, Ruth Van de Water and Yuzhi Liu for thoughtful comments on the manuscript. R. Z. was partially supported by NSF Grant PHY-1212389 and DOE Grant FG02-91ER 40661. Fermilab is operated by Fermi Research Alliance, LLC, under Contract No. DEAC02- 07CH11359 with the U.S. Department of Energy. S. G. was supported by DOE Grants FG02-91ER 40661 and DE-SC0010120.

\section{References}

[1] T. Blum, R. Zhou, T. Doi, M. Hayakawa, T. Izubuchi, S. Uno and N. Yamada, Phys. Rev. D 82, 094508 (2010) [arXiv:1006.1311 [hep-lat]].

[2] R. Horsley, et al. [QCDSF and UKQCD Collaborations], Phys. Rev. D 86, 114511 (2012) [arXiv:1206.3156 [hep-lat]].

[3] S. Basak, et al. [MILC Collaboration], PoS LATTICE 2012, 137 (2012) [arXiv:1210.8157 [hep-lat]].

[4] S. Basak, et al. [MILC Collaboration], PoS CD 12, 030 (2013) [arXiv:1301.7137 [hep-lat]].

[5] S. Borsanyi, et al. [BMW Collaboration], Phys. Rev. Lett. 111, 252001 (2013) [arXiv:1306.2287 [hep-lat]].

[6] G. M. de Divitiis, et al. [RM123 Collaboration], Phys. Rev. D 87, 114505 (2013) [arXiv:1303.4896 [hep-lat]].

[7] T. Ishikawa, T. Blum, M. Hayakawa, T. Izubuchi, C. Jung and R. Zhou, Phys. Rev. Lett. 109, 072002 (2012) [arXiv:1202.6018 [hep-lat]].

[8] S. Aoki, et al. [PACS-CS Collaboration], Phys. Rev. D 86, 034507 (2012) [arXiv:1205.2961 [hep-lat]].

[9] T. Blum, M. Hayakawa and T. Izubuchi, PoS LATTICE 2012, 022 (2012) [arXiv:1301.2607 [hep-lat]].

[10] R. Horsley, et. al. [QCDSF Collaboration], PoS LATTICE 2013, 499 (2013) [arXiv:1311.4554 [hep-lat]].

[11] S. Borsanyi, et al. [BMW Collaboration], arXiv:1406.4088 [hep-lat].

[12] A. Bazavov, D. Toussaint, C. Bernard, J. Laiho, C. DeTar, L. Levkova, M. B. Oktay, S. Gottlieb et al., Rev. Mod. Phys. 82, 1349 (2010) [arXiv:0903.3598 [hep-lat]].

[13] S. A. Gottlieb, W. Liu, D. Toussaint, R. L. Renken and R. L. Sugar, Phys. Rev. D 35, 2531 (1987).

[14] V. Azcoiti, A. Cruz, E. Dagotto, A. Moreo and A. Lugo, Phys. Lett. B 175, 202 (1986).

[15] J. Bijnens and N. Danielsson, Phys. Rev. D 75, 014505 (2007) [hep-lat/0610127]. 